\title{
MAPPING CYLINDERS AND THE ANNULUS CONJECTURE
}

\author{
BY L. S. HUSCH ${ }^{1}$
}

Communicated by R. H. Bing, October 14, 1968

Suppose $f$ is an embedding of the $n$-sphere $S^{n}$ into the $(n+1)$-sphere $S^{n+1}$; $f$ is said to be locally flat at $x \in S^{n}$ if there is a neighborhood $U$ of $f(x)$ in $S^{n+1}$ such that the pair $\left(U, U \cap f\left(S^{n}\right)\right)$ is homeomorphic to $\left(E^{n+1}, E^{n}\right)$ where $E^{i}$ is Euclidean $i$-space; i.e., there exists a homeomorphism $h: U \rightarrow E^{n+1}$ such that $h\left(U \cap f\left(S^{n}\right)\right)=E^{n} \equiv E^{n} \times 0 \subseteq E^{n} \times E^{1}$ $=E^{n+1}$. Brown [2], [3] has shown that if $f$ is locally flat at each point of $S^{n}$, then the closure of each complementary domain of $f\left(S^{n}\right)$ in $S^{n+1}$ is homeomorphic to an $(n+1)$-cell. One of the outstanding unsolved problems in topology of manifolds is the annulus conjecture. Suppose $f, g$ are two locally flat embeddings (i.e., $f$ and $g$ are locally flat at each point of $S^{n}$ ) of $S^{n}$ into $S^{n+1}$ such that $f\left(S^{n}\right) \cap g\left(S^{n}\right)=\varnothing$. The connected submanifold $A^{n+1}$ of $S^{n+1}$ whose boundary is $f\left(S^{n}\right)$ $\cup g\left(S^{n}\right)$ is called a pseudo-annulus. The annulus conjecture is that $A^{n+1}$ is homeomorphic to $S^{n} \times[0,1]$. If $f, g$ are both either piecewise linear or differentiable maps or if $n \leqq 2$, then the conjecture is true.

This paper was motivated by an attempt to construct a counterexample to the annulus conjecture. Let $p: S^{n} \rightarrow S^{n}$ be a continuous map. The mapping cylinder of $p$, Map $(p)$, is the decomposition space formed from the disjoint union $\left(S^{n} \times[0,1]\right) \cup S^{n}$ by identifying $(x, 1)$ with $p(x)$ for each $x \in S^{n}$. The idea was to find a map $p: S^{n} \rightarrow S^{n}$ such that Map $(p)$ is an $(n+1)$-manifold which is not homeomorphic to $S^{n} \times I$; for example, one might attempt to construct such a $p$ by using a variation of Bing's example [1] of an upper semicontinuous decomposition of $S^{3}$ which yields $S^{3}$ but some of whose nondegenerate elements are spheres. By Proposition 2, Map ( $p$ ) would be a pseudoannulus and hence a counterexample. However, we show that this is impossible in dimension 3; i.e., if $\operatorname{Map}(p)$ is a manifold, then it is homeomorphic to $S^{3} \times I$.

The author expresses his gratitude to Professor R. H. Bing who shortened many of the original arguments. Chris Lacher has obtained similar results.

Let $p: S^{n} \rightarrow S^{n}$ be a continuous map such that Map $(p)$ is an $(n+1)$ manifold. Let $\pi:\left(S^{n} \times I\right) \cup S^{n} \rightarrow$ Map $(p)$ be the natural projection.

${ }^{1}$ Research supported in part by National Science Foundation grant GP-8615. 
Proposition 1. The boundary of Map ( $p)$ is the union of the two $n$-spheres $\left(S^{n} \times 0\right)$ and $\left(S^{n} \times 1\right)$.

Proof. Suppose $M$ has one boundary component. Note that $M$ is homotopically equivalent to $S^{n}$. In the exact sequence

$$
H_{n}(\partial M) \stackrel{i_{*}}{\rightarrow} H_{n}(M) \rightarrow H_{n}(M, \partial M)
$$

$i_{*}$ is the zero map and by Poincare Duality, $H_{n}(M, \partial M)$ is isomorphic to $H^{1}(M)=0$. Hence $H_{n}(M)=0$, a contradiction.

Proposition 2. Map ( $p)$ is a pseudo-annulus.

Proof. By attaching an $(n+1)$-cell to each boundary component of $M$, one obtains a closed manifold $S$. It is easy to see that $S$ is the union of two open $(n+1)$-cells and hence by [2], $S$ is an $(n+1)$-sphere in which $M$ appears as a pseudo-annulus.

PRoposition 3. If $n \neq 4$, then $p$ is a cellular map; i.e., if $x \in S^{n}$, then $p^{-1}(x)=\bigcap_{i=1}^{\infty} C_{i}$ where $C_{i}\left(\subseteq\right.$ interior $\left.C_{i-1}\right)$ are closed $n$-cells in $S^{n}$.

Proof. Let $U$ be a contractible open subset of $S^{n}$. Define $g: p^{-1} U$ $\rightarrow U$ by $g=p \mid p^{-1} U$. Map $(g)$ is a contractible open subset of Map $(p)$ for Map $(g)=r^{-1} U$ where $r$ is the canonical deformation retraction of Map $(p)$ onto image $(p)=S^{n}$. Thus Map $(g)$ is an $(n+1)$-manifold. Since $U$ is collared in Map $(g)$ [3], Map $(g)-U$ is contractible. But Map $(g)-U$ deformation retracts to $p^{-1} U$ and hence $p^{-1} U$ is contractible. By Lacher [5, Theorem 2] for any open subset $V$ of $S^{n}, p: p^{-1}(V) \rightarrow V$ is a proper homotopy equivalence. Let $x \in S^{n}$, then $x=\bigcap_{i=1}^{\infty} D_{i}$ where $D_{i}\left(\subseteq\right.$ interior $D_{i-1}$ ) are closed $n$-cells in $S^{n}$. Since $p^{-1}(x)=\bigcap_{i=1}^{\infty} p^{-1} D_{i}$, if we want to show that $p^{-1}(x)$ is cellular, it is sufficient to show that there exists an $n$-cell $C_{i}$ in $p^{-1}$ (interior $D_{i}$ ) for each $i$ such that $p^{-1}(x)$ is contained in the interior of $C_{i}$. From above $p: p^{-1}$ (interior $D_{i}$ ) $\rightarrow$ interior $D_{i}$ is a proper homotopy equivalence; since interior $D_{i}$ is 1 -connected at infinity, $p^{-1}$ (interior $D_{i}$ ) is 1-connected at infinity. For $n=3, p^{-1}$ (interior $D_{i}$ ) is an open 3 -cell by Edwards [4]. For $n \geqq 5$, we apply Stallings [7]. It is now easy to find $C_{i}$.

TheOREM. If $p: S^{3} \rightarrow S^{3}$ is a continuous map and Map $(p)$ is a manifold, then Map $(p)$ is homeomorphic to $S^{3} \times I$.

Proof. By Proposition 3, $p$ is a cellular map. By Price [6] there exists a pseudo-isotopy $H: S^{3} \times I \rightarrow S^{3} \times I$ (i.e., $H$ is level preserving and the map $H_{t}: S^{3} \rightarrow S^{3}$, defined by $H(x, t)=\left(H_{t}(x), t\right)$, is a homeomorphism for $t \in[0,1)$ ) such that $H_{0}$ is the identity map and $H_{1}=p$. 
Define $\phi: \operatorname{Map}(p) \rightarrow S^{2} \times I$ by $H \pi^{-1}(x)$. It is easily seen that $\phi$ is a homeomorphism using the fact that $\pi$ is an open map.

\section{REFERENCES}

1. R. H. Bing, Decompositions of $E^{3}$, Topology of 3-manifolds and related topics (Proc. The University of Georgia Institute, 1961), Prentice-Hall, Englewood Cliffs, N. J., 1962, pp. 5-21.

2. M. Brown, A proof of the generalized Schoenfies theorem, Bull. Amer. Math. Soc. 66 (1960), 74-76.

3. Locally flat embeddings of topological manifolds, Ann. of Math. (2) 75 (1962), 331-341.

4. C. H. Edwards, Jr., Open 3-manifolds which are simply connected at infinity, Proc. Amer. Math. Soc. 14 (1963), 391-395.

5. R. C. Lacher, Cell-like mappings of ANR's, Bull. Amer. Math. Soc. 74 (1968), 933-935.

6. T. M. Price, Decompositions of $S^{3}$ and pseudo-isotopies, Notices Amer. Math. Soc. 15 (1968), 136.

7. J. Stallings, The piecewise linear structure of euclidean space, Proc. Cambridge Philos. Soc. 58 (1962), 481-489.

University of Georgia, Athens, Georgia 30602 